OPEN ACCESS

Edited by:

Xiangming $\mathrm{He}$,

Tsinghua University, China

Reviewed by:

Nitash P. Balsara

University of California, Berkeley,

United States

Zonghai Chen,

Argonne National Laboratory (DOE),

United States

Peng Bai,

Washington University in St. Louis,

United States

*Correspondence:

Didier Devaux

didier.devaux@grenoble-inp.fr

Renaud Bouchet

renaud.bouchet@grenoble-inp.fr

Specialty section:

This article was submitted to Electrochemical Energy Conversion and Storage,

a section of the journal

Frontiers in Energy Research

Received: 14 September 2019 Accepted: 24 December 2019

Published: 22 January 2020

Citation:

Devaux D, Leduc H, Dumaz P,

Lecuyer $M$, Deschamps $M$ and Bouchet R (2020) Effect of Electrode and Electrolyte Thicknesses on All-Solid-State Battery Performance Analyzed With the Sand Equation.

Front. Energy Res. 7:168

doi: 10.3389/fenrg.2019.00168

\section{Effect of Electrode and Electrolyte Thicknesses on All-Solid-State Battery Performance Analyzed With the Sand Equation}

\author{
Didier Devaux ${ }^{1 *}$, Hugo Leduc ${ }^{1}$, Philippe Dumaz ${ }^{1}$, Margaux Lecuyer ${ }^{2}$, Marc Deschamps $^{2}$ \\ and Renaud Bouchet ${ }^{1 *}$ \\ ${ }^{1}$ Univ. Grenoble Alpes, Univ. Savoie Mont Blanc, CNRS, Grenoble INP, LEPMI, Grenoble, France, ${ }^{2}$ Blue Solutions, Odet, \\ Ergué Gabéric, Quimper, France
}

The energy conversion and storage are great challenges for our society. Despite the progress accomplished by the Lithium(Li)-ion technology based on flammable liquid electrolyte, their intrinsic instability is the strong safety issue for large scale applications. The use of solid polymer electrolytes (SPEs) is an adequate solution in terms of safety and energy density. To increase the energy density (resp. specific energy) of the batteries, the positive electrode thickness must be augmented. However, as for Li-ion liquid electrolyte, the cationic transference number of SPEs is low, typically below 0.2, which limits their power performance because of the formation of strong gradient of concentration throughout the battery. Thus, for a given battery system a compromise between the energy density and the power has to be found in a rapid manner. The goal of this study is to propose a simple efficient methodology to optimize the thickness of the SPE and the positive electrode based on charge transport parameters, which allows to determine the effective limiting $\mathrm{Li}^{+}$diffusion coefficient. First, we rapidly establish the battery power performance thanks to a specific discharge protocol. Then, by using an approach based on the Sand equation a limiting current density is determined. A unique mother curve of the capacity as a function of the limiting current density is obtained whatever the electrode and electrolyte thicknesses. Finally, the effective limiting diffusion coefficient is estimated which in turn allows to design the best electrode depending on electrolyte thickness.

Keywords: lithium battery, polymer electrolyte, power performance, transport limitation, effective diffusion coefficient, power cycling procedure

\section{INTRODUCTION}

Batteries are one of the most widely used electrochemical energy storage devices thanks to their high energy permitting to operate devices for a long period of time (Kim et al., 2015). The Lithium (Li)-ion system is the predominant solution to power many applications from small electronic devices to electric cars (Kurzweil and Garche, 2017). As the demand in energy output from consumers is constantly increasing new battery systems must be developed and optimized depending on the application requirements. In this context $\mathrm{Li}$ metal is an ideal as negative electrode due to its high specific capacity, and low operating voltage (Xu et al., 2014). However, in combination with a conventional liquid electrolyte, the $\mathrm{Li}$ electrodeposits on the metallic $\mathrm{Li}$ are uneven giving rises 
to low Coulombic efficiency and dendrite growth (Tarascon and Armand, 2001; Wu et al., 2019). Replacing the liquid electrolyte by a solid polymer electrolyte (SPE) permits to envision safe high energy density batteries (Armand, 1994; Agrawal and Pandey, 2008).

In term of design, a practical Li metal battery stack should be optimized through its positive electrode formulation and overall material assembly notably to reduce the cost per elemental cell (Gallagher et al., 2014). The goal being to produce a long lasting battery with high rate performance to obtain extended specific energy (Eftekhari, 2017; Schnell et al., 2018; Zeng et al., 2019). Regarding the SPE, enough ionic conductivity in the order of $1 \mathrm{mS} . \mathrm{cm}^{-1}$ is obtained at $80^{\circ} \mathrm{C}$ for PEO-based electrolyte (Devaux et al., 2012). PEO possesses mechanical properties and flexibility high enough to be processed by hot-pressing, extrusion, or solvent-casting methods to form of thin films ranging from 10 to $100 \mu \mathrm{m}$ (Baudry et al., 1997; Porcarelli et al., 2016; Schnell et al., 2018; Yang et al., 2019). The goals being to minimize the SPE thickness to reduce ohmic loss and to increase the diffusion limited current density during battery operation. Moreover, PEO possesses other advantages such as a good adhesion, and electrochemical and chemical stability toward metallic Li (Armand, 1983). Indeed, PEO is usually used as a fundamental SPE brick which will also contain other polymers or additives to improve the desired properties-transport number, mechanical strength, electrochemical window, etc. (Bouchet et al., 2013; Zhang et al., 2017). $\mathrm{LiFePO}_{4}$ is a reference candidate as positive active materials thanks to its bi-phased redox reaction at about $3.43 \mathrm{~V}$ vs. $\mathrm{Li}^{+} / \mathrm{Li}^{\circ}$ which ensures safety (Padhi et al., 1997). At last, for a given electrode formulation, the energy density is directly linked to the active material loading (Du et al., 2017). So, for a given active material, increasing the electrode thickness is the simplest way to reach higher specific energy.

The electrode formulation is of importance through the loading of active material and the electrode porosity to reach the best power performance at an optimal electrode thickness (Newman, 1995; Yu et al., 2006, 2013). Zheng et al. reported on positive electrodes made of $\mathrm{LiNi}_{1 / 3} \mathrm{Co}_{1 / 3} \mathrm{Mn}_{1 / 3} \mathrm{O}_{2}$ (NCM) or $\mathrm{LiFePO}_{4}$ and showed that $\mathrm{Li}$ ion diffusion within the electrolyte in the electrode was the limiting discharge process (Zheng et al., 2012). Interestingly, they showed that the specific $C$-rate at which capacity starts to drop is representative to the overall battery rate performance. The electrode capacity, and thus active material loading, follows a negative power law, with the $C$-rate. Therefore, as expected low loaded electrodes provide higher rate performance than their higher loaded counterparts. A similar conclusion was substantially drawn by Gallagher et al. who combined experimental and simulation approaches and Heubner et al. on thick NCM electrodes (Gallagher et al., 2016; Heubner et al., 2019). In consequence, the ionic diffusion processes at stake in a battery system must be fully characterized as it governs the battery voltage divergence before the full recovery of the capacity because the Li-ion concentration reaches a null concentration at the cathode. All other electrochemical processes (ion and electron migration, charge transfer, etc.) are mostly related to "ohmic" drops which simply increase the battery polarization when the rate increases.
Typically, the relationship between discharge capacity and $C$-rate is similar for each battery technology. At low $C$-rate, the capacity is maximal and constant. At $C$-rate higher than a threshold value, the capacity falls rapidly (Gallagher et al., 2016). This behavior limits the high rate capabilities in terms of rapid charge and fast discharge (Doyle and Newman, 1997; Kang and Ceder, 2009). In literature a series of empirical and semi-empirical equations based on a phenomenological stretched exponential decay function have been developed to fit the sigmoidal shape of the capacity-C/rate curve (Gallagher et al., 2016; Wong et al., 2017; Heubner et al., 2018b; Tian et al., 2019). The physical meaning behind these equations is to consider a time dependent relaxation process describing herein a single rate limiting diffusion phenomena. So, those equations use as main parameters (i) a time constant and (ii) an empirical exponent value to stretch the exponential function. The meaning of the exponent is unclear as some studies set this parameter to a constant value of 2 or let it free to get better fits. The uncertainty regarding this empirical parameter is similar to that encounter with the Peukert exponent (Doerffel and Sharkh, 2006).

Therefore, ionic diffusion in the electrolyte, and within the active materials are known to be the major physical limitations that affect the battery rate capability (Jiang and Peng, 2016). Thus by coupling experimental and simulation analysis, many studies aim to improve power and energy from the single cell to the stack level (Yu et al., 2006; Safari and Delacourt, 2011; Singh et al., 2015). The mostly accepted theoretical model is the one developed by Newman and coworker based on concentrated solution theory (Doyle et al., 1993; Fuller et al., 1994; Newman, 1995; Srinivasan and Newman, 2004). A complete description of a given battery system is required to fit the cycling data which requires numerous parameters. Some of these parameters such as transference number, ionic diffusion coefficients, thermodynamic factors or tortuosity, to cite a few, are difficult to obtain properly as their determination is rather tough and non-univocal which can lead to large approximation. In addition, each set of parameters has to be determined again each time a chemical modification is made (additives, binders, electrode formulation, active materials...) which can be time consuming.

In practice, the fast determination of the discharge capacity depending on the $C$-rate is of interest to screen series of batteries in which many parameters are tuned such as the porosity, the formulation, and the active material loading of electrodes or even the nature of the current collectors. Back in 1994, a protocol was proposed composed of successive discharges starting from the highest $C$-rate down to the lowest without charging step in between (Doyle et al., 1994). More recently, Heubner et al. presented a methodology based on chronoamperometry measurement (Heubner et al., 2018a). Starting from charged Liion batteries, instead of applying a constant current as discharge step a constant voltage step at the lower battery cut-off voltage is performed. The transitory current recorded is then converted through the current-time integration into relative capacity. This method, a slow charge plus the chronoamperometry, takes about a dozen of hours to complete, but presents some limitations to fully capture the battery capacity when the active materials go 
through different phase change in charge (resp. in discharge). As pointed out by the authors, a typical example of such material is $\mathrm{LiFePO}_{4}$ with a two-phase mechanism.

Herein, firstly, to reduce the time-consuming measures necessary to test battery power depending on all the parameters a cycling procedure similar to that of Doyle et al. is used (Doyle et al., 1994). Through this rapid cycling routine, the relationship between discharge capacity and current density is determined with a good accuracy at least 8 times faster than conventional galvanostatic charge-discharge cycles alternation. Then, we propose two complementary methodologies to quickly estimate the effective diffusion coefficient of the rate limiting process at stake which allows by comparing it to the diffusion coefficient into the electrolyte or active material particles, to identify which component can be optimized. Those methodologies are based on limiting current and Sand time (Sand, 1901; Bard and Faulkner, 2001). For this purpose model all-solid-state batteries made of Li metal, PEO based electrolyte acting as SPE, and LFP-based positive electrode were used. By keeping the negative electrode in excess, the interplay between positive electrode and SPE thicknesses is finely understood and general rules are provided to pinpoint toward the optimal balance in thicknesses between SPE and positive electrode. The goal being to provide a simple physical interpretation of practical power signature curves based on the Sand's equation, which permits the determination of the effective limiting diffusion processes in real system.

\section{EXPERIMENTAL}

The metallic Li foil was provided by the company Blue Solutions. The $\mathrm{Li}$ was stored in an Argon filled glove box (Campus, Jacomex) with sub-ppm values of $\mathrm{H}_{2} \mathrm{O}$ and $\mathrm{O}_{2}$. The solid polymer electrolyte (SPE) is a random copolymer comprising $\mathrm{PEO}$ to ensure ionic motion and poly(propylene oxide) to provide flexibility to the resulting membrane, and doped with lithium bistrifluoromethanesulfonimide (LiTFSI) salt. The concentration of Li salt was added to reach a molar ratio of ethylene oxide to $\mathrm{Li}$ salt of 25. The Li salt and Li metal foil are stored in an Argon filled glove box (Campus, Jacomex) with sub ppm value of $\mathrm{H}_{2} \mathrm{O}$ and $\mathrm{O}_{2}$. The polymer and LiTFSI were dissolved in Dimethylformamide (DMF) by stirring at $350 \mathrm{rpm}$ and $80^{\circ} \mathrm{C}$ for $3 \mathrm{~h}$ in a vial. When the solution was clear and transparent, it was cast onto Petri dish made of Teflon and let dry at ambient temperature $(T)$ for $8 \mathrm{~h}$. Then, to remove the solvent, the Petri dish was placed in an oven at $60^{\circ} \mathrm{C}$ for at least 3 days. The resulting SPE membrane was peeled off the Teflon substrate and placed inside the Ar glove box for at least a week prior any further manipulation. Still inside the glove box, SPEs were cut into wide pieces and several of them placed in a hot press. The SPEs were then pressed for several minutes à $80^{\circ} \mathrm{C}$ at 200 bars while adjusting the SPE quantity. After pressing and cooling, SPE disks were punch out from the pressed SPE pieces. This lead to SPE disk with thickness $(y)$ of 18 , $36,54,108$, and $216 \mu \mathrm{m}$. Low porosity $(<5 \%)$ positive electrodes using $\mathrm{LiFeO}_{4}$ as active materials were specifically prepared by Blue Solutions through an extrusion process. The thickness of the positive electrode $(x)$ were $20,33,48$, and $60 \mu \mathrm{m}$ on top of an $\mathrm{Al}$ current collector. The capacities of the electrodes are proportional to their thicknesses.

Inside the globe box, a Li disk of $14 \mathrm{~mm}$ diameter was punch out from the Li foil. Then, a layer of SPE was also cut in a disk shape of $16 \mathrm{~mm}$ in diameter. The two materials were laminated at $80^{\circ} \mathrm{C}$ and 3 bars multiple times using a homemade laminating machine until the SPE was fully adherent to the Li. The electrolyte thickness was checked after the laminating process and no variation was observed. A $12 \mathrm{~mm}$ diameter positive electrode disk was also punch out from the electrode foil. Several passages through the laminating machine were done to ensure adhesion between the three material layers composing the Li metal polymer battery. The battery assemblies were varied in terms of positive electrode and SPE thicknesses. So, all along the text, the Li Metal Polymer (LMP) batteries are denoted $\operatorname{LMP}(x-y)$ with $x$ and $y$ corresponding to the electrode and SPE thicknesses, respectively. For each $\operatorname{LMP}(x-y)$ system, between 4 and 6 replicates were assembled.

After assembly, the battery was placed inside a stainless steel CR2032 coin cell using stainless steel shims and a wave spring. The coin cell was sealed using a crimping machine inside the glove box. On average 1.2 bars are applied to the battery assembly with an active surface $S$ corresponding to the positive electrode geometrical surface. Then, the battery cells were taken out of the glove box and placed on coin cell holder. The coin cells were put inside an oven (Memmert) held at $80^{\circ} \mathrm{C}$ and connected to a VMP3 (Bio-Logic SAS) multi-potentiostat with impedance capability.

The cycling procedure consists in a series of charge-discharge galvanostatic cycles in between 2.5 and $3.7 \mathrm{~V}$ vs. $\mathrm{Li}^{+} / \mathrm{Li}^{\circ}$. All along the text the battery potential $E$ is referred to the $\mathrm{Li}^{+} / \mathrm{Li}^{\circ}$ couple. Initially, the batteries are subjected to 8 conditioning cycles at a low current density $\left(J_{0}\right)$ identical in charge and discharge so that the delivered specific capacity corresponds to the effective $\mathrm{LiFePO}_{4}$ capacity, $160 \mathrm{mAh} . \mathrm{g}^{-1}$. After this initial procedure, conventional galvanostatic cycling to produce the power signature of the battery is made. It consists in a series of subsequent charge and discharge steps by constantly charging at a current density of $J_{0}$ and by increasing the discharge current density $J_{\mathrm{n}}$. In between each cycle charge/discharge a rest period of $30 \mathrm{~min}$ is used to relax the gradients of concentration. Taking into account the rest periods, this conventional cycling procedure needs more than a week-long time period. At each step, the areal charge capacity $\left(Q_{n}\right)$ was calculated by integration of the current density $J_{\mathrm{n}}$ over time $(t)$ during the galvanostatic steps according to:

$$
Q_{\mathrm{n}}=\int J_{\mathrm{n}}(t) \cdot d t
$$

For the rapid power procedure, after the conditioning galvanostatic cycles at $J_{0}$ previously described, the batteries are fully charged also at $J_{0}$, then we accumulate several discharges separated by a rest period of $30 \mathrm{~min}$ to relax the gradients of concentration. We start by the highest current density, followed by $30 \mathrm{~min}$ of relaxation, then a discharge at slightly lower current density is performed, and so on, until a 
final discharge step at $J_{0}$ (the lowest current density). This is done without charging the battery in between each discharge steps. For clarity, the cycling behavior, evolution of $E$ as a function of the fraction $\delta$ of $\mathrm{Li}$ inserted in the $\mathrm{Li}_{\delta} \mathrm{FePO}_{4}$ phase, with 0 $<\delta<1$, for a representative $\operatorname{LMP}(48-18)$ battery is provided in Supplementary Figure 1. This rapid power procedure takes about 1 day to fully complete from the charge to the final discharge step, at least eight times shorter than the conventional cycling procedure. The underlying assumption of this cycling procedure is that by starting from a fully charged battery, the discharge capacity at a given current density $J_{\mathrm{n}}$ is the sum of the discharge capacities obtained at higher current densities, $J>J_{\mathrm{n}}$, plus the one obtained when applying $J_{\mathrm{n}}$. In other word, $Q_{\mathrm{n}}\left(J_{\mathrm{n}}\right)$ is calculated based on the following equation:

$$
Q_{\mathrm{n}}=\sum_{N \geq n}\left[J_{\mathrm{N}} \cdot \Delta t_{\mathrm{N}}\right]
$$

with $\Delta t_{\mathrm{N}}$ the time necessary to discharge the battery under the constant current density $J_{\mathrm{N}}$.

For both cycling procedures, the capacities calculated at each $J_{\mathrm{n}}$ of the battery replicates lie within a typical deviation below $1 \%$ which shows the very good reproducibility of our cells. This is a mandatory condition to go further into the data analysis.

Finally, to have an independent measure of the transport properties of our electrolyte, electrochemical impedance spectroscopy was executed on Li symmetric cell comprising the SPE (Bouchet et al., 2003). This technic permits to determine the different cell resistances such as the electronic $\left(R_{\mathrm{c}}\right)$, electrolyte $\left(R_{\mathrm{el}}\right)$, interface $\left(R_{\mathrm{int}}\right)$, and diffusion $\left(R_{\mathrm{d}}\right)$ resistances. Li symmetric cells were assembled via a similar lamination process than that described for the LMP batteries and sealed in CR2032 coin cells. After placing the cells in an oven at $80^{\circ} \mathrm{C}$, impedance spectroscopy using an excitation signal of $40 \mathrm{mV}$ in a frequency range between $10 \mathrm{MHz}$ and $0.1 \mathrm{~Hz}$ was done. A typical Nyquist plot showing the opposite of the impedance imaginary part $[-\operatorname{Im}(Z)]$ as a function of the real part $[\operatorname{Re}(Z)]$ is presented in Supplementary Figure 2. An electrical equivalent circuit, displays in the inset of Supplementary Figure 2, comprising the cell resistances $\left(R_{\mathrm{c}}, R_{\mathrm{el}}, R_{\text {int }}\right)$, a cable inductance $\left(L_{\mathrm{c}}\right)$ at high frequency, a constant phase element for the interface $\left(C P E_{\text {int }}\right)$, and a short Warburg element $\left(W_{\mathrm{d}}\right)$ for the diffusion loop at low frequencies, permits to model the Nyquist plot. From the Warburg element two main parameters are extracted corresponding to $R_{\mathrm{d}}$ and the relaxation time $\left(\tau_{\mathrm{r}}\right)$ at the maximum of the quarter of lemniscate. $R_{\mathrm{d}}$ and $R_{\mathrm{el}}$ are related to the cationic transference number $\left(t^{+}\right)$while $\tau_{\mathrm{r}}$ is linked to the ambipolar diffusion coefficient $\left(D_{\mathrm{amb}}\right)$ and the SPE thickness $y$ by the following equations (Sørensen and Jacobsen, 1982; Ross MacDonald, 1992; Bouchet et al., 2003):

$$
\begin{gathered}
t^{+}=\frac{R_{\mathrm{el}}}{R_{\mathrm{el}}+R_{\mathrm{d}}} \\
\tau_{\mathrm{r}}=2.54 \cdot \frac{\left(\frac{y}{2}\right)^{2}}{D_{\mathrm{amb}}}
\end{gathered}
$$

In addition, $D_{\mathrm{amb}}$ is linked to the $\mathrm{Li}^{+}$diffusion coefficient $\left(D_{\mathrm{Li}^{+}}\right)$ by the relationship.

$$
D_{\mathrm{Li}^{+}}=\frac{D_{\mathrm{amb}}}{2 \cdot\left(1-t^{+}\right)}
$$

\section{RESULTS AND DISCUSSION}

The cycling behavior of a typical $\operatorname{LMP}(48-18)$ battery is shown in Figure 1 that represents $E$ as a function of the fraction $\delta$ of $\mathrm{Li}$ inserted in the $\mathrm{Li}_{\delta} \mathrm{FePO}_{4}$ phase, with $0<\delta<$ 1 , recorded during conventional cycling. For clarity, only a representative charge performed at $J_{0}=0.1 \mathrm{~mA} . \mathrm{cm}^{-2}$ is represented in Figure 1 and some discharge current density are indicated. The battery charge presents a typical long plateau at about $3.44 \mathrm{~V}$ corresponding to the oxidation of LFP materials (Padhi et al., 1997) followed by a potentiostatic step at $3.7 \mathrm{~V}$ to reach the full electrode capacity. During discharge, a lower potential plateau at about $3.40 \mathrm{~V}$, corresponding to the reduction of LFP, is observed. This plateau is less pronounced for discharge steps executed at $J_{\mathrm{n}}$ higher than $0.3 \mathrm{~mA} . \mathrm{cm}^{-2}$ due to an increase in the battery of gradient of concentration. Moreover, these observations on the conventional cycling behavior of a typical $\operatorname{LMP}(48-18)$ battery remain valid for all of the other considered $\operatorname{LMP}(x-y)$ batteries and associated replicates.

For the conventional cycling (see Figure 1) and the rapid power test (see Supplementary Figure 1), the discharge capacities were extracted by using Equations (1) and (2), respectively. Then, each $Q_{n}$ value was normalized by the discharge capacity delivered at $J_{0}$, denoted $Q_{0}$. Thus, Figure 2 represents the normalized discharge capacity, ratio $Q_{n} / Q_{0}$, as a function of the discharge current densities, $J_{n}$, for the two

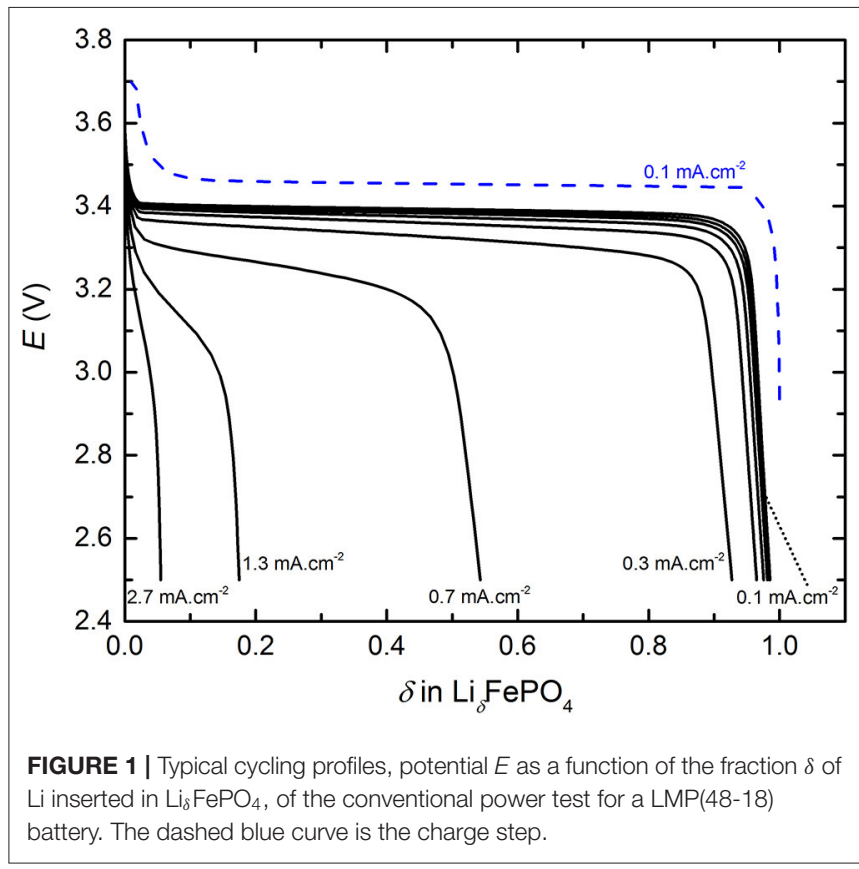




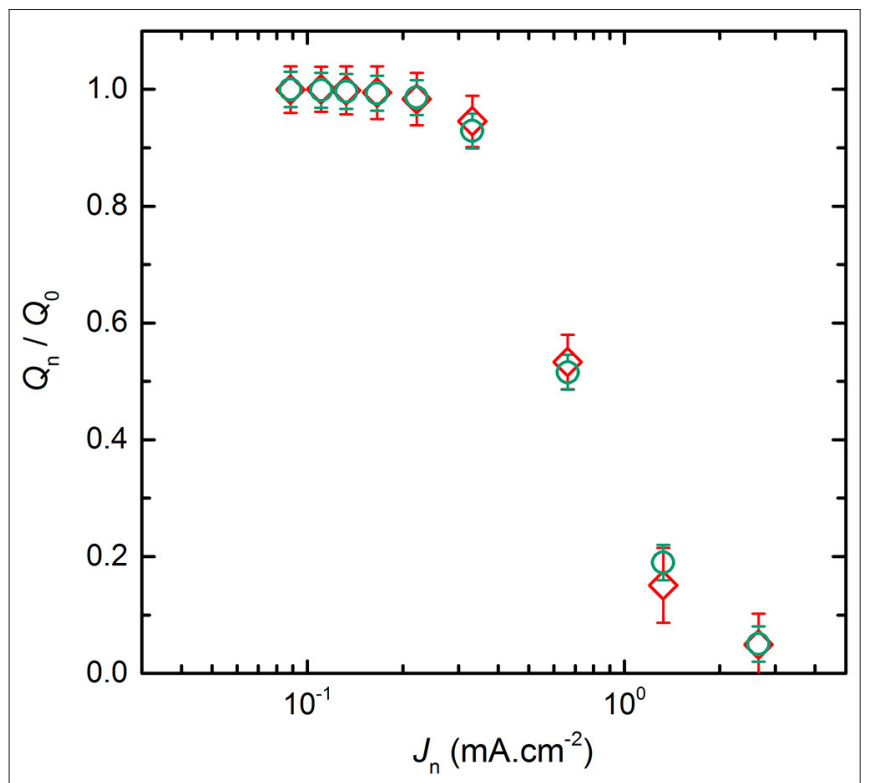

FIGURE 2 | Average normalized capacity, ratio $Q_{n} / Q_{0}$, of the $L M P(48-18)$ battery as a function of the discharge current densities, $J_{n}$, for the two cycling procedures. The symbols correspond to the $(\diamond)$ conventional cycling and $(\bigcirc)$ rapid power test.

cycling procedures of the $\operatorname{LMP}(48-18)$ batteries. The values reported in Figure 2 corresponds to average values with their standard deviations from the different battery replicates. For both procedures and for low $J_{\mathrm{n}}$ values, below $0.3 \mathrm{~mA} . \mathrm{cm}^{-2}, Q_{\mathrm{n}}$ remains close to $Q_{0}$ by $3 \%$. For both procedures and for high $J_{\mathrm{n}}$ values, higher than $0.3 \mathrm{~mA} . \mathrm{cm}^{-2}$, the ratio $Q_{\mathrm{n}} / Q_{0}$ quickly drops linearly with the increase of $J_{\mathrm{n}}$ down to values below 0.2 when $J_{\mathrm{n}}>1 \mathrm{~mA} \cdot \mathrm{cm}^{-2}$. Similarly to Li-ion batteries (Gallagher et al., 2014), the relationship between the discharge capacity and the current density of the all-solid-state Li batteries present a transition regime at a critical current density value. Moreover, the data point recorded at the highest $J_{\mathrm{n}}$ value, at $2.8 \mathrm{~mA} . \mathrm{cm}^{-2}$, deviates from the linear trend of $Q_{\mathrm{n}} / Q_{0}$ with $J_{\mathrm{n}}$ when $J_{\mathrm{n}}>$ $0.3 \mathrm{~mA} . \mathrm{cm}^{-2}$ due to supercapacitor effect arising from the high surface carbon fillers and coating of the LFP particles. Such capacitive effect is only seen for the highest current densities of the $\operatorname{LMP}(x-y)$ batteries and is not taken into account for the data analysis provided in the remaining of the text. The evolution of the normalized discharge capacities with the current densities are in excellent accordance for the conventional cycling and the rapid power test. Indeed, differences in $Q_{n} / Q_{0}$ values lie within the error bars typically when $J_{\mathrm{d}}>0.3 \mathrm{~mA} . \mathrm{cm}^{-2}$. In addition, the accordance in between the two procedures is also observed for each studied $\operatorname{LMP}(x-y)$ batteries. The rapid power test is then a robust tool to picture the battery power performance in a time-efficient manner with a strong accuracy when compared to conventional cycling procedure. Other interesting cycling routine can be found in literature but will less accuracy regarding phase transition positive active materials as LFP (Heubner et al., 2018a).

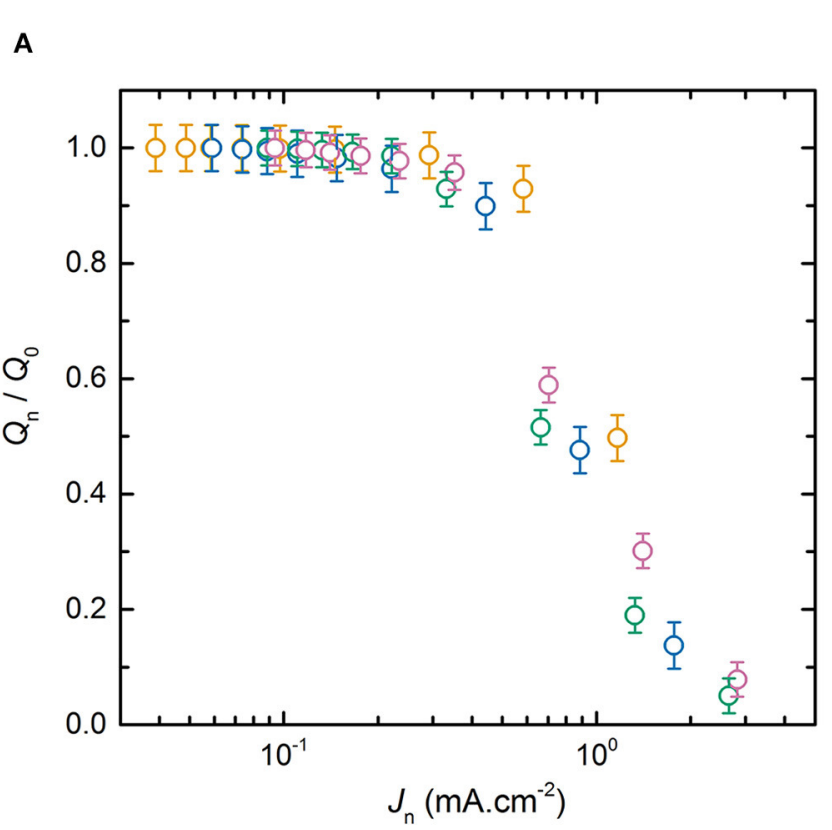

B

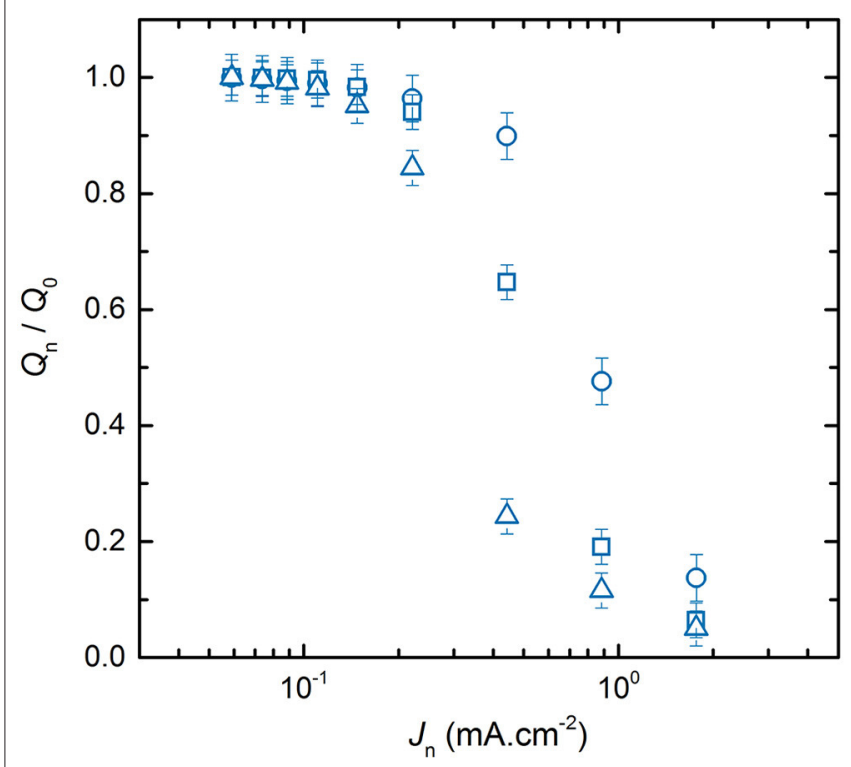

FIGURE 3 | Average normalized capacity, ratio $Q_{n} / Q_{0}$, as a function of the discharge current densities $J_{n}$. (A) $\operatorname{LMP}(x-18)$ batteries with positive electrode thickness $x$ of (orange) 20, (blue) 33, (green) 48, and (pink) $60 \mu \mathrm{m}$; and (B) LMP(33-y) batteries with SPE thickness $y$ of $(\bigcirc) 18$, (口) 36, and $(\Delta) 54 \mu \mathrm{m}$.

To understand the effect of electrode thicknesses on battery power, Figure $\mathbf{3 A}$ represents the average normalized discharge capacity as a function of $J_{\mathrm{n}}$ for the $\operatorname{LMP}(x-18)$ batteries with $x$ values of $20,33,48$, and $60 \mu \mathrm{m}$ while the SPE thickness is maintained at $18 \mu \mathrm{m}$. For each $\operatorname{LMP}(x-y)$ batteries reported in Figure 3A, the evolution of $Q_{n} / Q_{0}$ with $J_{d}$ is similar to that already detailed in Figure 2. The main difference between 
each battery is the location of the transition regime between the current density regime where $Q_{n}$ remains close to $Q_{0}$ and the regime where $Q_{\mathrm{n}} / Q_{0}$ decreases with $J_{\mathrm{d}}$. Based on Figure $3 \mathrm{~A}$, the thinnest the positive electrode, the later $Q_{\mathrm{n}}$ will deviate strongly from $Q_{0}$. A similar conclusion can be drawn for all the other $\operatorname{LMP}(x-y)$ batteries in which $y$ is constant and $x$ being either 20,33,48, or $60 \mu \mathrm{m}$. For completeness, Supplementary Figure 3 shows the ratio $Q_{n} / Q_{0}$ as a function of $J_{\mathrm{n}}$ for the $\operatorname{LMP}(x-36)$ and $\operatorname{LMP}(x-54)$ batteries. The effect of the positive electrode thickness on the power is then similar to that reported for Li-ion batteries comprising a liquid electrolyte (Cornut et al., 2015; Heubner et al., 2018b). As a complementary case, Figure 3B shows the average normalized discharge capacity as a function of $J_{\mathrm{n}}$ for the $\operatorname{LMP}(33-y)$ batteries with $y$ values of 18,36 , and $54 \mu \mathrm{m}$ while the positive electrode thickness is constant at $33 \mu \mathrm{m}$. For a given positive electrode thickness, the transition regime occurs at lower current density when the SPE thickness increases. This observation remains valid for all the other $\operatorname{LMP}(x-y)$ batteries in which $x$ is constant and $y$ being either 18, 36, 54, 108, and $216 \mu \mathrm{m}$. For completeness, Supplementary Figure 4 shows the ratio $Q_{n} / Q_{0}$ as a function of $J_{\mathrm{n}}$ for the $\operatorname{LMP}(20-y), \operatorname{LMP}(48-y)$, and $\operatorname{LMP}(60-y)$. From Figures $3 \mathbf{A}, \mathbf{B}$, the power performance of the all-solid-state $\mathrm{Li}$ batteries are dependent on both positive electrode and SPE thicknesses. Interestingly, in most of the studies on standard Li-ion batteries, only the electrode thickness has been varied shading the impact of the electrolyte thickness. Understanding finely the partition of these two parameters on the battery performance is of first interest to envision an optimized battery assembly.

From each $Q_{n} / Q_{0}$ vs. $J_{n}$ plots, we define the current density corresponding to the transition regime as the current density at which the $Q_{n} / Q_{0}=1$ baseline (low $J_{n}$ regime) crosses the linear interpolation of $Q_{\mathrm{n}} / Q_{0} v s$. $J_{\mathrm{n}}$ (high $J_{\mathrm{n}}$ regime). This drop of capacity is due to a $\mathrm{Li}$ ion diffusion limitation, therefore current density corresponding to this data point is called the diffusion limiting current, denoted $J_{\text {lim }}$. For clarity, the graphical determination of $J_{\text {lim }}$ is shown in Supplementary Figure 5 for the LMP(20-18) batteries. In order to provide more insight on $J_{\text {lim }}$, we propose a methodology based on the Sand equation for controlled-current technique (Sand, 1901; Rosso et al., 2006). By definition, for a current density above $J_{\text {lim }}$, the Sand time $\left(\tau_{\mathrm{s}}\right)$ corresponds to the transition time at which the flux of ionic species is not large enough to satisfy the applied current. The Sand equation (Brissot et al., 1999) is theoretically given by:

$$
\tau_{\mathrm{s}}=\pi \cdot D_{\mathrm{amb}} \cdot\left(\frac{n \cdot F \cdot C_{\mathrm{Li}}}{2 \cdot\left(1-t^{+}\right) \cdot J_{\mathrm{n}}}\right)^{2}
$$

with $n$ the number of exchanged electron ( $n=1$ for LFP), $F$ the Faraday constant $\left(9.64810^{4} \mathrm{C} . \mathrm{mol}^{-1}\right), C_{\mathrm{Li}}$ the Li concentration in the SPE (Devaux et al., 2012) located in the electrolyte and in the positive electrode $\left(882 \mathrm{~mol} . \mathrm{m}^{-3}\right.$ for both), $t^{+}$the cationic transference number, and $D_{\mathrm{amb}}$ the ambipolar diffusion coefficient. The use of this equation is an approximation since the boundaries condition of a plan electrode is not perfectly respected on the cathode side.
For $J_{\mathrm{n}} \geq J_{\text {lim }}, \tau_{\mathrm{s}}$ can be estimated as being equivalent to the discharge time. In other words, $\tau_{\mathrm{s}}$ at a specific current density is defined as:

$$
\tau_{\mathrm{s}}\left(J_{\mathrm{n}}\right)=\frac{Q_{\mathrm{n}}}{J_{\mathrm{n}}} \text { when } J_{\mathrm{n}} \geq J_{\lim }
$$

$D_{\text {amb }}$ and $t^{+}$of a Li salt in PEO can be measured or calculated using many techniques such as electrochemical methodologies based on polarization (Shi and Vincent, 1993; Geiculescu et al., 2006) or relaxation (Mullin et al., 2011), impedance spectroscopy (Bouchet et al., 2003), pulse-field NMR (Hayamizu et al., 1999), or molecular dynamic simulation (Diddens et al., 2010) to name a few. By looking into literature data and focusing on high molecular PEO based electrolyte, at $80^{\circ} \mathrm{C}, D_{\mathrm{amb}}$ is in the range of $510^{-8} \mathrm{~cm}^{2} \cdot \mathrm{s}^{-1}$ and $t^{+}$of about 0.15 . In addition, impedance spectroscopy was performed on Li symmetric cells comprising SPE thickness of 18,36 , and $54 \mu \mathrm{m}$. The electrical equivalent circuit (see inset of Supplementary Figure 2) was used to fit all the impedance spectra in order to extract $t^{+}$and $D_{\text {amb }}$ values. $D_{L i^{+}}$, calculated using Equation (5), and $t^{+}$are both independent of the SPE thickness with an average value consistent with literature analysis of $3.4 \pm 0.710^{-8} \mathrm{~cm}^{2} . \mathrm{s}^{-1}$ and $0.15 \pm 0.02$, respectively.

The plot of $\tau_{\mathrm{s}}$ as a function of $J_{\mathrm{n}}^{-2}$ shows a linear behavior for the data points for which the condition $J_{\mathrm{n}} \geq J_{\lim }$ is satisfied which confirms the Sand behavior. As an example, Supplementary Figure 6 shows $\tau_{\mathrm{s}}$ vs. $J_{\mathrm{n}}^{-2}$ for a $\operatorname{LMP}(48-18)$ battery including the linear regression line. The slope of the regression line is then directly proportional to $D_{\mathrm{amb}}$ and thus to $D_{\mathrm{Li}^{+}}$. Considering a $t^{+}$of 0.15 as determined by impedance spectroscopy, $D_{L^{+}}$has been calculated for each $\operatorname{LMP}(x-y)$ battery. $D_{L^{+}}$is independent of the positive electrode and SPE thickness with an average value of $3.1 \pm 0.610^{-8} \mathrm{~cm}^{2} . \mathrm{s}^{-1}$. So, the $\mathrm{Li}^{+}$diffusion coefficient determined by the Sand equation when applied to battery cycling data is rather similar to the $\mathrm{Li}^{+}$diffusion coefficient within the SPE. Therefore, the power limiting phenomena in these all-solid-state batteries is then the diffusion of the $\mathrm{Li}^{+}$cation in the electrolyte spanning from the $\mathrm{Li}$ negative electrode to the $\mathrm{Al}$ current collector of the positive electrode rather than the diffusion of $\mathrm{Li}^{+}$in the LFP active material (Doyle and Newman, 1995). The use of the sand equation is then an efficient tool to quickly determine the effective limiting diffusion process at stake in batteries when $J>J_{\text {lim }}$, i.e., at rates where only a part of the full capacity is recovered.

To go further and compare all the LMP $(x-y)$ batteries together, $J_{\lim }$ was extracted from each $Q_{\mathrm{n}} / Q_{0}$ vs. $J_{n}$ plots. Figure 4 represents the average normalized capacity as a function of the ratio $J_{\lim } / J_{\mathrm{n}}$ for the $\operatorname{LMP}(20-18), \operatorname{LMP}(33-36)$, LMP(48-216), and LMP(60-54) batteries. All the experimental normalized capacity curves of the $\operatorname{LMP}(x-y)$ batteries overlap to a mere curve which demonstrates that the limiting phenomenon in stake here is the same whatever the electrode and electrolyte thickness and is due to the diffusion in the polymer electrolyte. In literature, a mere curve is usually obtained when the normalized capacity is represented as a function of the $C$-rate or its inverse. The mere curve is then fitted by an exponential decay function stretched by 


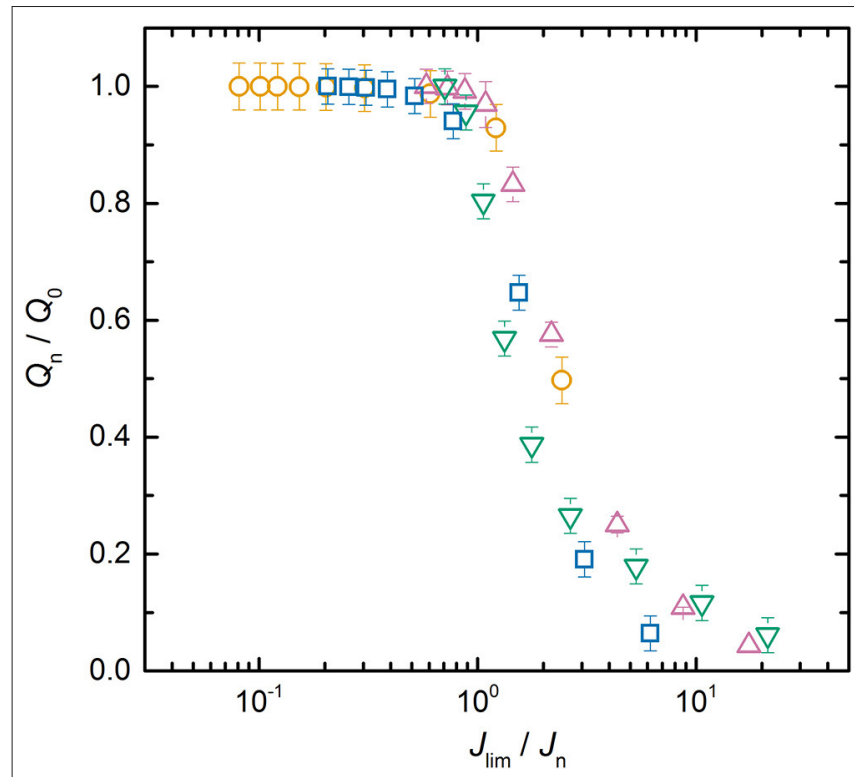

FIGURE 4 | Average normalized capacity, ratio $Q_{n} / Q_{0}$, as a function of $J_{\text {lim }} / J_{n}$ for the (○) LMP(20-18), ( $\square$ ) LMP(33-36), ( $\nabla) \operatorname{LMP}(48-36)$, and ( $\triangle$ ) LMP(60-54) batteries. an empirical value, typically of 2, and adjusted using a relaxation time parameter (Heubner et al., 2018b; Tian et al., 2019). In our case, no semi-empirical function is used as the only parameter corresponds to a physical parameter $J_{\text {lim }}$, graphically determined. The next step is to provide a simple model to get more insight on the partition of $J_{\text {lim }}$ due to the diffusion into the electrolyte and/or into the electrolyte spanning into the positive electrode.

Figure 5 represents the average $J_{\text {lim }}$ of the different $\operatorname{LMP}(x-$ $y$ ) batteries as a function of the positive electrode thickness $x$. For a given positive electrode thickness, $J_{\lim }$ increases with the diminution of the SPE thickness. The $J_{\text {lim }}$ values span within those obtained for a $20 \mu \mathrm{m}$ thick positive electrode between 0.06 and $0.48 \mathrm{~mA} . \mathrm{cm}^{-2}$ when $y$ equals to 216 and $18 \mu \mathrm{m}$, respectively. When $y=18 \mu \mathrm{m}, J_{\text {lim }}$ decreases linearly with $x$. For higher $y$ value, the slope of the $J_{\text {lim }}$ decays with $x$ is less pronounced as $y$ increases until a plateau value for the highest reported SPE thicknesses of $216 \mu \mathrm{m}$. The best power performance is thus expected for battery comprising a thin positive electrode and a thin SPE layer. However, by looking at the interplay between the $x$ and $y$ value, battery with higher specific energy can be assembled without impairing much the power performances. Indeed, $J_{\lim }$ is an indicator of the onset of battery capacity decrease from its nominal value, i.e., $Q_{0}$. In Figure 5, the LMP(60-18) battery shows a $J_{\lim }$ value higher than the one of the LMP(20-36). This implies that $J_{\text {lim }}$ is a weak function of the positive electrode thickness and a strong function of the SPE thickness. In consequence, for the $\operatorname{LMP}(x-y)$ batteries, the specific volumetric energy density per single cell can be augmented simply by selecting the best compromise between positive electrode and SPE thicknesses.

The weak dependence of $J_{\lim }$ relatively to $x$ can be understood by considering that the effective surface ensuring the $\mathrm{Li}^{+}$diffusive

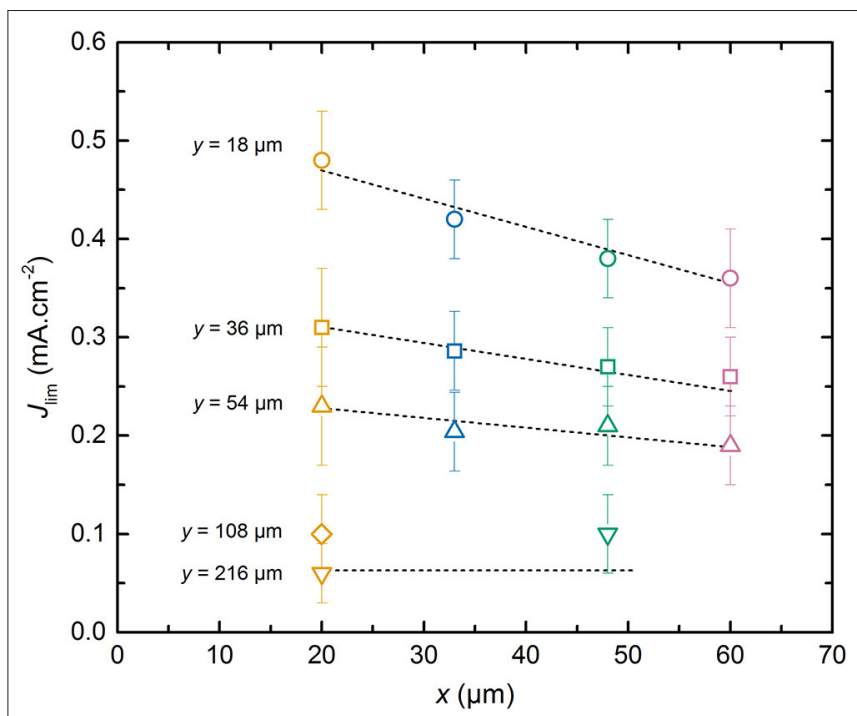

FIGURE 5 | Average limiting current, J lim, as a function of the positive electrode thickness, $x$. The dashed lines are guidelines for the eyes depending on the SPE thickness, $y$. The symbols correspond to SPE thickness $y$ of $(\bigcirc)$ $18,(\square) 36,(\triangle) 54,(\diamond) 108$, and $(\nabla) 216 \mu \mathrm{m}$. The symbol colors correspond to positive electrode thickness $x$ of (orange) 20, (blue) 33, (green) 48, and (pink) $60 \mu \mathrm{m}$.

flux at the interface of composite electrode/electrolyte is lower than the geometrical surface of the electrode due to the presence of active material and carbon particles in the composite electrode. So, the current density corresponding to the flux of $\mathrm{Li}^{+}$in the SPE located inside the electrode is higher than the one in the electrolyte. In addition, the $\mathrm{Li}^{+}$diffusion length depends on the electrode tortuosity. These effects can be described by introducing a parameter $\alpha$ with $0<\alpha \leq 1$ in which the total $\mathrm{Li}^{+}$ diffusion path corresponds to $\alpha . x+y$. One can expect that the diffusion path in the composite electrode should be longer than the thickness of the electrode due to its tortuosity. However, here we measure an effective value, and it appears that the diffusion in the electrolyte located in the electrode seems higher than that of the raw electrolyte probably due to moisture content. This leads to an $\alpha$ value below unity. In Figure 6, $J_{\lim }$ is plotted as a function of $\alpha . x+y$. By adjusting $\alpha$ to a value of 0.35 , all the $J_{\lim }$ data collapse onto a master curve that is fitted using a reciprocal function, shown as the dashed curve in Figure 6, that yields to the following equation:

$$
J_{\lim }=\frac{K}{(\alpha \cdot x+y)}
$$

with $K=13.2 \mathrm{~mA} . \mathrm{cm}^{-1}$ based on least squares fit $\left(R^{2}>0.99\right)$.

To provide a physical meaning of the parameter $K$ in Equation (8), the $\operatorname{LMP}(x-y)$ can be modeled via a one dimensional approach as extensively performed by the group of Doyle et al. (1993). Thus, the Fick law in 1-D can be simply applied in which the diffusion current is proportional to the $\mathrm{Li}^{+}$concentration gradient, the thickness of the diffusion process, and the diffusion coefficient of the limiting phenomena. Based on the results 


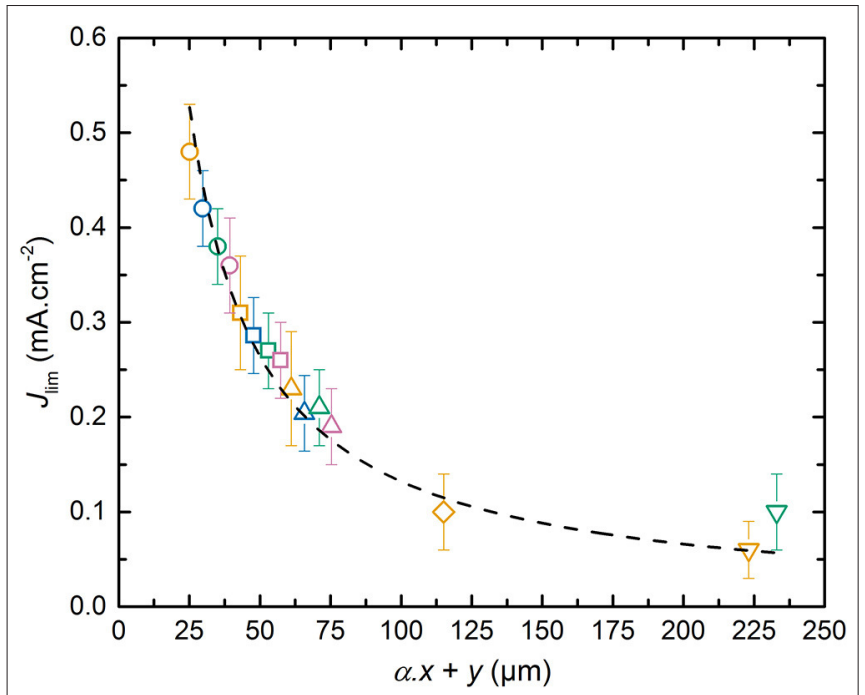

FIGURE 6 | Limiting current density, $J_{\text {lim }}$, as a function of $\alpha . x+y$. The dashed curve corresponds to the fit obtained using a reciprocal function. The symbols correspond to SPE thickness $y$ of $(\bigcirc) 18,(\square) 36,(\triangle) 54,(\diamond) 108$, and $(\nabla)$ $216 \mu \mathrm{m}$. The symbol colors correspond to positive electrode thickness $x$ of (orange) 20, (blue) 33, (green) 48, and (pink) $60 \mu \mathrm{m}$.

obtained in Figure 6, $J_{\text {lim }}$ is an inverse function of the sum $\alpha$. $x+y$ and corresponds thus to the current value at which the $\mathrm{Li}$ concentration gradient throughout the battery falls down to a null value at the $\mathrm{Al}$ current collector/positive electrode. $J_{\lim }$ can then be expressed as a function of the effective $\mathrm{Li}^{+}$diffusion coefficient $\left(D_{L i}^{e f f}\right)$ according to :

$$
J_{\lim }=n \cdot F \cdot D_{L i}^{e f f} \cdot\left(\frac{C_{\mathrm{Li}}}{\alpha \cdot x+y}\right)
$$

Combining Equation (9) into (8) permits to directly calculate a corresponding $D_{L i}^{e f f}$ value of $1.610^{-8} \mathrm{~cm}^{2} . \mathrm{s}^{-1}$ based on the $K$ parameter. The effective diffusion coefficient is then in the same range than the $\mathrm{Li}^{+}$diffusion coefficient determined by the Sand time methodology. In consequence, the effective diffusion coefficient $D_{L i}^{\text {eff }}$ corresponds to the effective $\mathrm{Li}^{+}$diffusion coefficient $D_{\mathrm{Li}^{+}}$in the SPE from the positive electrode to electrolyte layer. The $J_{\lim }$ methodology is less accurate than the Sand time methodology but is much faster to implement in order to quickly determine the limiting diffusion phenomena in the batteries. In addition, the $\alpha$ parameter is interesting since it depends on the electrode formulation and tortuosity as well as the ionic transport within the SPE used in the positive electrode. The variability of this parameters as function of the electrode formulation is a tool to optimize the electrode composition and texture, but is beyond the scope of our study.

\section{CONCLUSION}

The battery power signature is rapidly determined via a rapid power test. This method consists in applying consecutive galvanostatic discharge step from high to low current density. The results given by this method is identical to that obtained by conventional galvanostatic cycling, i.e., series of charge-discharge steps. At current density higher than the limiting current density $\left(J_{\lim }\right)$, the application of the Sand equation for controlled-current technique is proven to be effective in determining the diffusion coefficient of the limiting process. Herein, in the Li polymer batteries, the $\mathrm{Li}^{+}$diffusion in the solid polymer electrolyte acting as battery separator and positive electrode binder limits the battery performance. In addition to the fast determination of $J_{\text {lim }}$, a physical meaning of this parameter is provided. $J_{\text {lim }}$ is directly linked to an effective $\mathrm{Li}^{+}$diffusion coefficient throughout the battery that is similar to the one reported by the Sand equation. In consequence, the rapid power test is an efficient technique to compare series of batteries that differ by their assembly and to determine the main limiting factor, and thus optimize in turn the battery assembly. Battery design as then to consider the cumulative effect of both effect arising from the SPE layer thickness and from the positive electrode thickness. For example, in our LMP batteries, for a $54 \mu \mathrm{m}$ thick electrolyte a positive electrode up to $48 \mu \mathrm{m}$ can be used without impairing the battery power performance. For completeness, other factors than the power performance should also be considered, especially in case of Li metal based battery with the impact of the heterogeneous Li electrodeposition during charge step. To go further, this methodology should be applied to Li metal polymer batteries having different positive electrode formulations as well as on other battery technology.

\section{DATA AVAILABILITY STATEMENT}

All datasets generated for this study are included in the article/Supplementary Material.

\section{AUTHOR CONTRIBUTIONS}

$\mathrm{RB}, \mathrm{DD}, \mathrm{MD}$, and $\mathrm{ML}$ designed the project study. $\mathrm{MD}$ and ML produced the composite electrodes. $\mathrm{DD}, \mathrm{PD}$, and $\mathrm{HL}$ conducted the experiments. RB, DD, and HL analyzed the data. $\mathrm{RB}$ and $\mathrm{DD}$ wrote the manuscript. All the authors commented the manuscript.

\section{FUNDING}

The work has been done in the context of a French FUI20 fund (Fond Unique Interministériel) with the collaborative project named ALEPH.

\section{ACKNOWLEDGMENTS}

We thank the BPI (Banque Publique d'Investissement) and the Blue Solutions company for their financial support.

\section{SUPPLEMENTARY MATERIAL}

The Supplementary Material for this article can be found online at: https://www.frontiersin.org/article/10.3389/fenrg. 2019.00168/full\#supplementary-material 


\section{REFERENCES}

Agrawal, R. C., and Pandey, G. P. (2008). Solid polymer electrolytes: materials designing and all-solid-state battery applications: an overview. J. Phys. D Appl. Phys. 41:223001. doi: 10.1088/0022-3727/41/22/223001

Armand, M. (1983). Polymer solid electrolytes - an overview. Solid State Ionics 9-10, 745-754. doi: 10.1016/0167-2738(83)90083-8

Armand, M. (1994). The history of polymer electrolytes. Solid State Ionics 69, 309-319. doi: 10.1016/0167-2738(94)90419-7

Bard, A. J., and Faulkner, L. R. (2001). Electrochemical Methods Fundamentals and Applications, 2nd Edn. New York, NY: John Wiley Sons, Inc.

Baudry, P., Lascaud, S., Majastre, H., and Bloch, D. (1997). Lithium polymer battery development for electric vehicle application. J. Power Sour. 68, 432-435. doi: 10.1016/S0378-7753(97)02646-3

Bouchet, R., Lascaud, S., and Rosso, M. (2003). An EIS study of the anode Li/PEO-LiTFSI of a Li polymer battery. J. Electrochem. Soc. 150, A1385-A1389. doi: $10.1149 / 1.1609997$

Bouchet, R., Maria, S., Meziane, R., Aboulaich, A., Lienafa, L., Bonnet, J.-P., et al. (2013). Single-ion BAB triblock copolymers as highly efficient electrolytes for lithium-metal batteries. Nat. Mater. 12, 452-457. doi: 10.1038/nmat3602

Brissot, C., Rosso, M., Chazalviel, J.-N., and Lascaud, S. (1999). Dendritic growth mechanisms in lithium/polymer cells. J. Power Sour. 81-82, 925-929. doi: 10.1016/S0378-7753(98)00242-0

Cornut, R., Lepage, D., and Schougaard, S. B. (2015). Interpreting lithium batteries discharge curves for easy identification of the origin of performance limitations. Electrochim. Acta 162, 271-274. doi: 10.1016/j.electacta.2014.11.035

Devaux, D., Bouchet, R., Glé, D., and Denoyel, R. (2012). Mechanism of ion transport in PEO/LiTFSI complexes: effect of temperature, molecular weight and end groups. Solid State Ionics 227, 119-127. doi: 10.1016/j.ssi.2012.09.020

Diddens, D., Heuer, A., and Borodin, O. (2010). Understanding the lithium transport within a rouse-based model for a PEO/LiTFSI polymer electrolyte. Macromolecules 43, 2028-2036. doi: 10.1021/ma901893h

Doerffel, D., and Sharkh, S. A. (2006). A critical review of using the Peukert equation for determining the remaining capacity of lead-acid and lithium-ion batteries. J. Power Sour. 155, 395-400. doi: 10.1016/j.jpowsour.2005.04.030

Doyle, M., Fuller, T., and Newman, J. (1993). Modeling of galvanostatic charge and discharge of the lithium/ polymer/insertion cell. J. Electrochem. Soc. 6, 1526-1533. doi: 10.1149/1.2221597

Doyle, M., and Newman, J. (1995). The use of mathematical modeling in the design of lithium/polymer battery systems. Electrochim. Acta 40, 2191-2196. doi: 10.1016/0013-4686(95)00162-8

Doyle, M., and Newman, J. (1997). Analysis of capacity-rate data for lithium batteries using simplified models of the discharge process. J. Appl. Electrochem. 27, 846-856. doi: 10.1023/A:1018481030499

Doyle, M., Newman, J., and Reimers, J. (1994). A quick method of measuring the capacity versus discharge rate for a dual lithium-ion insertion cell undergoing cycling. J. Power Sources 52, 211-216. doi: 10.1016/0378-7753(94)02012-4

Du, Z., Wood, D. L., Daniel, C., Kalnaus, S., and Li, J. (2017). Understanding limiting factors in thick electrode performance as applied to high energy density Li-ion batteries. J. Appl. Electrochem. 47, 405-415. doi: 10.1007/s10800-017-1047-4

Eftekhari, A. (2017). Lithium-ion batteries with high rate capabilities. ACS Sustain. Chem. Eng. 5, 2799-2816. doi: 10.1021/acssuschemeng.7b00046

Fuller, T. F., Doyle, M., and Newman, J. (1994). Simulation and optimization of the dual lithium ion insertion cell. J. Electrochem. Soc. 141, 1-10. doi: $10.1149 / 1.2054684$

Gallagher, K. G., Goebel, S., Greszler, T., Mathias, M., Oelerich, W., Eroglu, D., et al. (2014). Quantifying the promise of lithium-air batteries for electric vehicles. Energy Environ. Sci. 7, 1555-1563. doi: 10.1039/c3ee43870h

Gallagher, K. G., Trask, S. E., Bauer, C., Woehrle, T., Lux, S. F., Tschech, M., et al. (2016). Optimizing areal capacities through understanding the limitations of lithium-ion electrodes. J. Electrochem. Soc. 163, A138-A149. doi: 10.1149/2.0321602jes

Geiculescu, O. E., Rajagopal, R., Creager, S. E., DesMarteau, D. D., Zhang, X. W., and Fedkiw, P. (2006). Transport properties of solid polymer electrolytes prepared from oligomeric fluorosulfonimide lithium salts dissolved in high molecular weight poly(ethylene oxide). J. Phys. Chem. B 110, 23130-23135. doi: $10.1021 /$ jp062648p
Hayamizu, K., Aihara, Y., Arai, S., and Martinez, C. G. (1999). Pulse-gradient spinecho $1 \mathrm{H}, 7 \mathrm{Li}$, and $19 \mathrm{~F}$ NMR diffusion and ionic conductivity measurements of 14 organic electrolytes containing $\mathrm{LiN}\left(\mathrm{SO}_{2} \mathrm{CF}_{3}\right)_{2}$. J. Phys. Chem. B 103 , 519-524. doi: 10.1021/jp9825664

Heubner, C., Lämmel, C., Nickol, A., Liebmann, T., Schneider, M., and Michaelis, A. (2018a). Comparison of chronoamperometric response and rate-performance of porous insertion electrodes: Towards an accelerated rate capability test. J. Power Sour. 397, 11-15. doi: 10.1016/j.jpowsour.2018. 06.087

Heubner, C., Nickol, A., Seeba, J., Reuber, S., Junker, N., Wolter, M., et al. (2019). Understanding thickness and porosity effects on the electrochemical performance of $\mathrm{LiNi}_{0.6} \mathrm{Co}_{0.2} \mathrm{Mn}_{0.2} \mathrm{O}_{2}$-based cathodes for high energy Liion batteries. J. Power Sour. 419, 119-126. doi: 10.1016/j.jpowsour.2019. 02.060

Heubner, C., Seeba, J., Liebmann, T., Nickol, A., Börner, S., Fritsch, M., et al. (2018b). Semi-empirical master curve concept describing the rate capability of lithium insertion electrodes. J. Power Sources 380, 83-91. doi: 10.1016/j.jpowsour.2018.01.077

Jiang, F., and Peng, P. (2016). Elucidating the performance limitations of lithiumion batteries due to species and charge transport through five characteristic parameters. Sci. Rep. 6:32639. doi: 10.1038/srep32639

Kang, B., and Ceder, G. (2009). Battery materials for ultrafast charging and discharging. Nature 458, 190-193. doi: 10.1038/nature07853

Kim, J. G., Son, B., Mukherjee, S., Schuppert, N., Bates, A., Kwon, O., et al. (2015). A review of lithium and non-lithium based solid state batteries. J. Power Sour. 282, 299-322. doi: 10.1016/j.jpowsour.2015.02.054

Kurzweil, P., and Garche, J. (2017). "Overview of batteries for future automobiles," in Lead-Acid Batteries for Future Automobiles, eds J. Garche, E. Karden, P. T. Moseley, and D. A. J. Rand (Amsterdam: Elsevier BV), 27-96. doi: 10.1016/B978-0-444-63700-0.00002-7

Mullin, S. A., Stone, G. M., Panday, A., and Balsara, N. P. (2011). Salt diffusion coefficients in block copolymer electrolytes. J. Electrochem. Soc. 158, A619A627. doi: $10.1149 / 1.3563802$

Newman, J. (1995). Optimization of porosity and thickness of a battery electrode by means of a reaction-zone model. J. Electrochem. Soc. 142, 97-101. doi: $10.1149 / 1.2043956$.

Padhi, A. K., Nanjundaswamy, K. S., and Goodenough, J. B. (1997). Phosphoolivines as positive-electrode materials for rechargeable lithium batteries. $J$. Electrochem. Soc. 144, 1188-1194. doi: 10.1149/1.1837571

Porcarelli, L., Gerbaldi, C., Bella, F., and Nair, J. R. (2016). Super soft all-ethylene oxide polymer electrolyte for safe all-solid lithium batteries. Sci. Rep. 6:19892. doi: $10.1038 /$ srep 19892 .

Ross MacDonald, J. (1992). Impedance/admittance response of a binary electrolyte. Electrochim. Acta 37, 1007-1014. doi: 10.1016/0013-4686(92)85216-8

Rosso, M., Brissot, C., Teyssot, A., Dollé, M., Sannier, L., Tarascon, J.-M., et al. (2006). Dendrite short-circuit and fuse effect on Li/Polymer/Li cells. Electrochim. Acta 51, 5334-5340. doi: 10.1016/j.electacta.2006.02.004

Safari, M., and Delacourt, C. (2011). Mathematical modeling of lithium iron phosphate electrode: galvanostatic charge/discharge and path dependence. J. Electrochem. Soc. 158, A63-A73. doi: 10.1149/1.3515902

Sand, H. J. S. (1901). On the concentration at the electrodes in a solution, with special reference to the liberation of hydrogen by electrolysis of a mixture of copper sulphate and sulphuric acid. Phil. Mag. 1, 45-79. doi: 10.1080/14786440109462590

Schnell, J., Günther, T., Knoche, T., Vieider, C., Köhler, L., Just, A., et al. (2018). All-solid-state lithium-ion and lithium metal batteries paving the way to large-scale production. J. Power Sour. 382, 160-175. doi: 10.1016/j.jpowsour.2018.02.062

Shi, J., and Vincent, C. A. (1993). The effect of molecular-weight on cation mobility in polymer electrolytes. Solid State Ionics 60, 11-17. doi: 10.1016/0167-2738(93)90268-8

Singh, M., Kaiser, J., and Hahn, H. (2015). Thick electrodes for high energy lithium ion batteries. J. Electrochem. Soc. 162, A1196-A1201. doi: 10.1149/2.0401507jes

Sørensen, P. R., and Jacobsen, T. (1982). Conductivity, chargetransfer and transport number - an ac-investigation of the polymer electrolyte LiSCN-poly(ethyleneoxide). Electrochim. Acta 27, 1671-1675. doi: 10.1016/0013-4686(82)80162-X 
Srinivasan, V., and Newman, J. (2004). Discharge model for the lithium iron-phosphate electrode. J. Electrochem. Soc. 151, A1517-A1529. doi: 10.1149/1.1785012

Tarascon, J.-M., and Armand, M. (2001). Issues and challenges facing rechargeable lithium batteries. Nature 414, 359-367. doi: 10.1038/35104644

Tian, R., Park, S.-H., King, P. J., Cunningham, G., Coelho, J., Nicolosi, V., et al. (2019). Quantifying the factors limiting rate performance in battery electrodes. Nat. Commun. 10:1933. doi: 10.1038/s41467-019-09792-9

Wong, L. L., Chen, H., and Adams, S. (2017). Design of fast ion conducting cathode materials for grid-scale sodium-ion batteries. Phys. Chem. Chem. Phys. 19, 7506-7523. d. doi: 10.1039/C7CP00037E

Wu, X., Song, K., Zhang, X., Hu, N., Li, L., Li, W., et al. (2019). safety issues in lithium ion batteries: materials and cell design. Front. Energy Res. 7:65. doi: 10.3389/fenrg.2019.00065

Xu, W., Wang, J., Ding, F., Chen, X., Nasybulin, E., Zhang, Y., et al. (2014). Lithium metal anodes for rechargeable batteries. Energy Environ. Sci. 7, 513-537. doi: 10.1039/C3EE40795K

Yang, J., Wang, X., Zhang, G., Ma, A., Chen, W., Shao, L., et al. (2019). High-performance solid composite polymer electrolyte for all solid-state lithium battery through facile microstructure regulation. Front. Chem. 7:388. doi: $10.3389 /$ fchem. 2019.00388

Yu, D. Y. W., Donoue, K., Inoue, T., Fujimoto, M., and Fujitani, S. (2006). Effect of electrode parameters on $\mathrm{LiFePO}_{4}$ cathodes. J. Electrochem. Soc. 153, A835-A839. doi: 10.1149/1.2179199

Yu, S., Kim, S., Kim, T. Y., Nam, J. H., and Cho, W. I. (2013). Model prediction and experiments for the electrode design optimization of $\mathrm{LiFePO}_{4} /$ Graphite electrodes in high capacity lithium-ion Batteries. Bull. Korean Chem. Soc. 34, 79-88. doi: 10.5012/bkcs.2013.34.1.79

Zeng, X., Li, M., Abd El-Hady, D., Alshitari, W., Al-Bogami, A. S., Lu, J., et al. (2019). Commercialization of lithium battery technologies for electric vehicles. Adv. Energy Mater. 9:1900161. doi: 10.1002/aenm.201900161

Zhang, H., Li, C., Piszcz, M., Coya, E., Rojo, T., Rodriguez-Martinez, L. M., et al. (2017). Single lithium-ion conducting solid polymer electrolytes: advances and perspectives. Chem. Soc. Rev. 46, 797-815. doi: 10.1039/C6CS00491A

Zheng, H., Li, J., Song, X., Liu, G., and Battaglia, V. S. (2012). A comprehensive understanding of electrode thickness effects on the electrochemical performances of Li-ion battery cathodes. Electrochim. Acta 71, 258-265. doi: 10.1016/j.electacta.2012.03.161

Conflict of Interest: MD and ML are employed by the company Blue Solutions.

The remaining authors declare that the research was conducted in the absence of any commercial or financial relationships that could be construed as a potential conflict of interest.

Copyright (c) 2020 Devaux, Leduc, Dumaz, Lecuyer, Deschamps and Bouchet. This is an open-access article distributed under the terms of the Creative Commons Attribution License (CC BY). The use, distribution or reproduction in other forums is permitted, provided the original author(s) and the copyright owner(s) are credited and that the original publication in this journal is cited, in accordance with accepted academic practice. No use, distribution or reproduction is permitted which does not comply with these terms. 